\title{
Quinidine Gluconate
}

National Cancer Institute

\section{Source}

National Cancer Institute. Quinidine Gluconate. NCI Thesaurus. Code C48015.

The gluconate salt form of quinidine, an alkaloid with antimalarial and antiarrhythmic

(Class la) properties. Quinidine gluconate exerts its anti-malarial activity by acting primarily as an intra-erythrocytic schizonticide through association with the hemepolymer (hemozoin) in the acidic food vacuole of the parasite thereby preventing further polymerization by heme polymerase enzyme. This results in accumulation of toxic heme and death of the parasite. Quinidine gluconate exerts its antiarrhythmic effects by depressing the flow of sodium ions into cells during phase 0 of the cardiac action potential, thereby slowing the impulse conduction through the atrioventricular (AV) node, reducing the maximal rate of phase 0 depolarization and prolong ing the refractory period. Quinidine gluconate also reduces the slope of phase 4 depolarization in Purkinjefibres resulting in slowed conduction and reduced automaticity in the heart. 